\title{
Microbiological surveillance and parenteral antibiotic use in a critical care unit
}

Sharon K Yamashita Pharm D ${ }^{1,2}$, Marie Louie MD FRCPC ${ }^{3,4}$, Andrew E Simor MD FRCPC ${ }^{3,4}$, Anita Rachlis MD FRCPC ${ }^{4}$

SK Yamashita, M Louie, AE Simor, A Rachlis. Microbiological surveillance and parenteral antibiotic use in a critical care unit. Can J Infect Dis 2000;11(2):107-111.

OBJECTIVE: To evaluate parenteral antibiotic utilization and bacterial resistance patterns in a critical care unit (CrCU). DESIGN: Descriptive, prospective audit of infection site, culture and antimicrobial susceptibility test results, parenteral antibiotic usage and duration, total antibiotic acquisition costs, and length of stay.

SETTING: A 17-bed medical-surgical CrCU in a tertiary care teaching hospital in Metropolitan Toronto.

PATIENTS: Two hundred and fifty-eight patients admitted to the CrCU between May 1995 and April 1996 who received antimicrobial therapy.

RESULTS: The most frequently prescribed antibiotics were cefazolin $(47 \%, 1098 \mathrm{~g})$, gentamicin $(33 \%, 141 \mathrm{~g})$ and ceftriaxone $(20 \%, 255 \mathrm{~g})$. The most common indications for antimicrobial therapy included surgical prophylaxis $(34 \%)$ and pneumonia (35\%). The following organisms were isolated from patients treated with antibiotics: Staphylococcus aureus (26\%), Pseudomonas aeruginosa (13\%), enterococci (12\%), Haemophilus influenzae (11\%), Escherichia coli (11\%), Enterobacter cloacae (8\%) and other Gram-negative bacilli (19\%). Only 9\% of Gram-negative bacilli were resistant to aminoglycosides, $3 \%$ were resistant to ciprofloxacin and no extended-spectrum beta-lactamases or imipenem-resistance were detected. No vancomycin-resistant enterococci and only two methicillin-resistant Staphylococcus aureus isolates were identified.

CONCLUSIONS: Antibiotic use during the audit appeared appropriate for the specific clinical indications. Low levels of bacterial resistance were detected during the audit.

Key Words: Antibiotic utilization; Bacterial resistance; Critical care unit

\section{Surveillance microbiologique et antibiothérapie par voie parentérale dans une unité de soins intensifs}

OBJECTIF: Évaluer l'utilisation de l'antibiothérapie par voie parentérale et la résistance bactérienne dans une unité de soins intensifs (USI).

PLAN D'ÉTUDE : Audit prospectif et descriptif du site d'infection, résultats des cultures et des tests de sensibilité antimicrobienne, utilisation et durée de l'antibiothérapie par voie parentérale, coûts totaux d'acquisition des antibiotiques et durée du séjour.

LIEU : USI médicaux et chirurgicaux, comptant 17 lits, dans un hôpital d'enseignement de soins tertiaires de la grande région de Toronto.

PATIENTS : Deux cent cinquante-huit patients admis à l'USI entre mai 1995 et avril 1996 et soumis à un traitement antimicrobien.

RÉSULTATS : Les antibiotiques prescrits le plus souvent étaient la céfazoline (47\%, 1098 g), la gentamicine (33\%,

voir page suivante

\footnotetext{
${ }^{1}$ Departments of Pharmacy, ${ }^{2}$ Clinical Pharmacology and ${ }^{3}$ Microbiology, and ${ }^{4}$ Division of Infectious Diseases, Department of Medicine, Sunnybrook and Women's College Health Sciences Centre, and University of Toronto, Toronto, Ontario

Correspondence: Dr Sharon Yamashita, Department of Pharmacy, Room E300, Sunnybrook Health Science Centre, 2075 Bayview Avenue,

Toronto, Ontario M4N 3M5. Telephone 416-480-4507, fax 416-480-5887, e-mail sharon.yamashita@swchsc.on.ca

Received for publication March 8, 1999. Accepted September 7, 1999
} 
$141 \mathrm{~g})$ et la ceftriaxone $(20 \%, 255 \mathrm{~g})$. Parmi les indications les plus courantes du traitement antimicrobien, il y avait la prophylaxie à des fins chirurgicales $(34 \%)$ et la pneumonie $(35 \%)$. On a isolé les organismes suivants chez les patients traités aux antibiotiques : Staphylococcus aureus (26\%), Pseudomonas aeruginosa (13\%), des entérocoques (12\%), Haemophilus influenzae (11\%), Escherichia coli (11\%), Enterobacter cloacae (8\%) et d'autres bacilles Gram négatif (19\%). Seulement $9 \%$ des bacilles Gram négatif étaient résistants aux aminosides, 3 \% à la ciprofloxacine, et on n'a pas noté de résistance aux pénicillinases à spectre élargi ou aux imipénèmes. Il n'y avait pas d'entérocoques résistants à la vancomycine, et seuls deux isolats de Staphylococcus aureus se sont avérés résistants à la méthicilline.

CONCLUSION : Les antibiotiques utilisés durant l'audit semblaient adaptés aux indications cliniques et on a observé un faible taux de résistance bactérienne.

$\mathrm{T}$ he emergence of antimicrobial resistance over the past several decades has challenged the management of infections in hospitalized patients, and has led to increased morbidity, higher health care costs and prolonged hospitalizations (1). Hospitals are now frequently encountering outbreaks of resistant organisms such as vancomycin-resistant enterococci (VRE) and methicillin-resistant Staphylococcus aureus (MRSA). Patients admitted to intensive care units, in particular, often become colonized with resistant organisms and may serve as the focus for hospital-wide bacterial resistance (2). The high prevalence of resistance in intensive care units (ICUs) has been attributed to the severity of illness of the patients, prolonged hospital stays, and the widespread use of invasive devices and broad spectrum antibiotics $(3,4)$.

It has been suggested that surveillance of bacterial resistance patterns is a useful technique to control the emergence of resistant organisms (5). Early detection of resistant organisms allows for specific measures to be implemented to prevent the widespread transmission of bacterial resistance. Furthermore, as the choice of antimicrobial therapy for nosocomial infections is often governed by local resistance patterns, surveillance of bacterial susceptibility can aid in making decisions regarding empirical antimicrobial therapy at each institution. The collection of information on antibiotic usage is also essential because selective pressure exerted by the use of certain antibiotics may impact on resistance patterns (6). The increased use of third-generation cephalosporins in the ICUs, for example, may lead to the selection of highly resistant Gram-negative bacilli $(7,8)$. Surveillance for both bacterial resistance and antimicrobial usage can therefore be used to improve antibiotic prescribing, as well as to detect early changes in resistance patterns that would require intervention.

We conducted a prospective one-year survey for the purpose of surveillance of antibiotic use and microbial resistance in our critical care unit ( $\mathrm{CrCU})$.

\section{PATIENTS AND METHODS}

At the time of the study, Sunnybrook Health Science Centre was a 982-bed (418 acute, 564 chronic), university-affiliated, adult tertiary care teaching hospital in Metropolitan Toronto. It is the largest level 1 trauma centre in Canada, with other major teaching programs that include aging, cardiovascular disease, oncology and trauma. At the time of the study, the $\mathrm{CrCU}$ was a 17 -bed unit that admitted medical, surgical and trauma patients, the majority of whom required mechanical ventilation.
Data were prospectively collected over a one-year period, May 1, 1995 to April 30, 1996, from all patients admitted to the $\mathrm{CrCU}$ for more than one day and who received parenteral antimicrobial therapy. Information collected by daily chart review included site of infection, culture and antimicrobial susceptibility test results and length of stay. Duration of parenteral antibiotic usage, as well as total antibiotic acquisition costs, were also recorded.

The diagnosis of infection and choice of antimicrobial therapy were at the discretion of the medical staff in the CrCU. There was, however, significant input into antimicrobial prescribing by the Departments of Pharmacy, Microbiology and Infectious Diseases. This included daily attendance at patient rounds by a clinical pharmacist and weekly rounds with a microbiologist. Table 1 includes all formulary antibiotics that were available for use in the CrCU. The medical staff in the $\mathrm{CrCU}$ was not aware that the audit was being performed.

Bacterial isolates were from all body sites (both sterile and nonsterile) and data recorded on these isolates were collected once the diagnosis of infection was made by the $\mathrm{CrCU}$ staff. No standard definitions of infection were used. Table 2 indicates the categories of infections as used by the medical staff in the $\mathrm{CrCU}$. Bacteremias were recorded as primary (no obvious source) or secondary.

Susceptibility testing of microorganisms was done according to recommended National Committee for Clinical Laboratory Standards (NCCLS) guidelines (9). The automated Vitek bacteriology system (bioMerieux Vitek Inc, Hazelwood, Missouri) was used for the identification of microorganisms and susceptibility testing. Detection of MRSA was confirmed using an oxacillin screen plate containing 6 g oxacillin and 4\% sodium chloride. VRE were detected according to the method of Jorgensen et al (10), using $6 \mathrm{~g}$ of vancomycin (Sigma Chemical Company, St Louis, Missouri) in brain-heart infusion agar (Difco Laboratories, Detroit, Michigan). Detection of extended-spectrum beta-lactamases (ESßL) was made only on Escherichia coli and klebsiella strains isolated from sterile body fluids, using the E-test (CAB Biodisk, Solna, Sweden) strips with a combination of a beta-lactam and beta-lactamase inhibitor.

Data collected from patients were entered and analyzed using Filemaker Pro (Claris Corporation, Santa Clara, California). Descriptive statistics were performed on the data presented. Length of stay data were reported as mean standard deviation. A Student's $t$-test was used to compare the length of stay of patients who received antibiotics with all patients admitted to the $\mathrm{CrCU}$ during the period of audit. 
TABLE 1

Parenteral antibiotic usage in the critical care unit at Sunnybrook Health Science Centre, Toronto, Ontario from May 1, 1995 to April 30, 1996*

\begin{tabular}{|c|c|c|c|c|}
\hline Antibiotic & $\begin{array}{l}\text { Number of patients }(\%) \\
\text { receiving antibiotic }(n=258)\end{array}$ & Average g/patient day & Total use $(g)^{+}$ & Total cost $(\$)^{\ddagger}$ \\
\hline \multicolumn{5}{|l|}{ Penicillins } \\
\hline Penicillin & $10(3.9)$ & 10.9 units & 906 units & 322.80 \\
\hline Ampicillin & $37(14.3)$ & 2.1 & 1028 & 686.40 \\
\hline Cloxacillin & $43(16.7)$ & 3.1 & 1913 & 1161.25 \\
\hline Piperacillin & $28(10.9)$ & 5.4 & 2879 & 8990.00 \\
\hline \multicolumn{5}{|l|}{ Cephalosporins } \\
\hline Cefazolin & $120(46.5)$ & 1.8 & 1098 & 2709.46 \\
\hline Cefuroxime & $41(15.9)$ & 1.1 & 323 & 3116.67 \\
\hline Cefotetan & $14(5.4)$ & 0.8 & 58 & 840.00 \\
\hline Ceftriaxone & $52(20.2)$ & 0.7 & 255 & 8634.00 \\
\hline Ceftazidime & $24(9.3)$ & 0.8 & 265 & 4028.00 \\
\hline \multicolumn{5}{|l|}{ Carbapenems } \\
\hline Imipenem & 0 & 0 & 0 & 0 \\
\hline \multicolumn{5}{|l|}{ Aminoglycosides } \\
\hline Gentamicin & $86(33.3)$ & 0.2 & 141 & 5088.46 \\
\hline Tobramycin & $32(12.4)$ & 0.1 & 81 & 6025.44 \\
\hline Amikacin & $1(0.4)$ & 0.05 & 2.5 & 146.25 \\
\hline \multicolumn{5}{|l|}{ Quinolones } \\
\hline Ciprofloxacin & $20(7.8)$ & 0.3 & 49 & 4384.02 \\
\hline \multicolumn{5}{|l|}{ Miscellaneous } \\
\hline Erythromycin & $19(7.4)$ & 1.7 & 178 & 1052.25 \\
\hline Vancomycin & $38(14.7)$ & 0.5 & 256 & 5370.36 \\
\hline Clindamycin & 48 (18.6) & 1.0 & 459 & 3666.05 \\
\hline Metronidazole & 49 (19) & 0.6 & 209 & 589.36 \\
\hline $\begin{array}{l}\text { Trimethoprim/ } \\
\text { sulphamethoxazole }\end{array}$ & $17(6.6)$ & $6.5 \mathrm{~mL}$ & $1590 \mathrm{~mL}$ & 1113.00 \\
\hline
\end{tabular}

\section{RESULTS}

There were 759 admissions to the $\mathrm{CrCU}$ during the study. One-third of patients were admitted from the emergency department, $32 \%$ of patients came from the operating room and $35 \%$ were transferred to the $\mathrm{CrCU}$ from another ward or hospital. A total of 258 (32\%) patients received parenteral antimicrobial therapy during their CrCU stay, including 75 medical, 105 surgical and 78 trauma patients. There were 340 courses of antibiotics administered; 226 were for treatment of infection and 114 were for surgical prophylaxis. The average duration of antimicrobial therapy for all patients included in the audit was 6.7 days (range one to 54 days). The most frequently prescribed antibiotic was cefazolin (47\% of patients, $1098 \mathrm{~g}$ total use), followed by gentamicin $(33 \%, 141 \mathrm{~g})$, ceftriaxone $(20 \%, 255 \mathrm{~g})$, metronidazole $(19 \%, 209 \mathrm{~g})$, clindamycin (19\%, 459 g) (Dalacin C, Pharmacia \& Upjohn Inc, Mississauga, Ontario), cloxacillin (17\%, $1913 \mathrm{~g})$, cefuroxime (16\%, 323 g) (Zinacef, Glaxo Canada Inc, Toronto, Ontario), vancomycin $(15 \%, 256$ g) (Vancocin, Eli Lilly Canada Inc, Scarborough, Ontario), ampicillin (14\%, $1028 \mathrm{~g})$ and tobramycin $(12 \%, 81 \mathrm{~g})$ (Table 1$)$. The overall cost of all parenteral antibiotics during the audit was $\$ 57,854.35$, which represented $18 \%$ of the total drug budget for the $\mathrm{CrCU}$ for that year.

The average length of stay for patients receiving antibiotics (10.7 \pm 14.7 days) was significantly higher than the length of
TABLE 2

Indications for antimicrobial therapy*

\begin{tabular}{lc}
\hline Indication & Number (\%) \\
\hline Pneumonia & $121(35.6)$ \\
Prophylaxis & $114(33.5)$ \\
Bacteremia & $30(8.8)$ \\
Primary & $20(5.9)$ \\
Secondary & $10(2.9)$ \\
Intra-abdominal infection & $23(6.8)$ \\
Skin/soft tissue infection & $12(3.5)$ \\
Urinary tract infection & $10(2.9)$ \\
Line-associated infection & $5(1.5)$ \\
Surgical wound infection & $4(1.2)$ \\
Other (sinusitis, meningitis, endocarditis, & $21(6.2)$ \\
mediastinitis, pseudomembranous colitis, & \\
febrile neutropenia) & \\
*As determined by the attending medical staff &
\end{tabular}

stay of all patients admitted to the $\mathrm{CrCU}$ ( $4.7 \pm 8.0$ days) during this period $(\mathrm{P}<0.01)$. There were 6.3 infections treated with antibiotics/100 patient days during the surveillance period. Of the patients who received antimicrobial therapy, $76 \%$ remained in the $\mathrm{CrCU}$ for more than $48 \mathrm{~h}$.

When the prophylactic antibiotic use was excluded from the analysis, both the average duration of antibiotic therapy 
and length of stay increased from 6.7 and 10.7 days to 8.5 (range one to 54) and 13.4 (range one to 146) days, respectively. There did not, however, appear to be a significant change in the most frequently prescribed antibiotics.

The indications for antimicrobial therapy as determined by the attending medical staff in the $\mathrm{CrCU}$ are as summarized in Table 2. Several patients had more than one infectious diagnosis. In $34 \%$ of the infections treated, no bacterial pathogen was identified. Of the culture results collected, $26.3 \%$ were from sterile sites. Of the 30 patients with positive blood cultures, 20 patients had a primary bacteremia without a clearly identified source. In addition to the blood cultures, only 11 other cultures were from sterile sites (eg, cerebrospinal fluid [CSF], tissue biopsies, specimens obtained during surgical procedures). Pneumonia accounted for over one-third of the indications for antimicrobial treatment. Of the pneumonias diagnosed on clinical and radiological features $(n=121)$, microbiological cultures were obtained from endotracheal tube specimens in all but seven; these seven samples were obtained from bronchoalveolar lavage specimens.

The most common organisms isolated from patients with infection were $S$ aureus (25.6\%), followed by Pseudomonas aeruginosa (13.5\%), Enterococcus species (12.2\%), Haemophilus influenzae (10.9\%), E coli (10.9\%), Enterobacter cloacae (7.7\%), other Gram-negative (12.8\%) and other Gram-positive organisms (4.5\%). In particular, $S$ aureus was the most frequently cultured organism (24.5\%) from blood, followed by Enterococcus species (13.2\%), Pseudomonas species (11.3\%), Staphylococcus epidermidis (9.4\%) and Candida albicans (9.4\%).

of all the $S$ aureus isolates recovered from patients who received parenteral antibiotics in the $\mathrm{CrCU}$, only $5 \%$ of the $S$ aureus strains were methicillin-resistant. There were no VRE or enterococci with high-level aminoglycoside resistance. ESßL or imipenem resistance were not found in Gram-negative bacilli. Twenty-nine per cent of the $H$ influenzae isolates were beta-lactamase positive. $E$ coli was found to have the highest antimicrobial resistance, with $41 \%$ resistant to ampicillin, $24 \%$ resistant to gentamicin and $6 \%$ resistant to cefazolin. For $E$ coli isolates collected from across the hospital during 1995 to 1996, the antimicrobial resistance rates were similar for ampicillin and cefazolin but not for gentamicin, for which the rate was only $4 \%$. Within the Enterobacteriaceae, there was no resistance to trimethoprim/sulphamethoxazole; one Klebsiella species was resistant to cefazolin; two Citrobacter freundii were resistant to gentamicin, with one also resistant to ciprofloxacin (Cipro, Bayer Inc, Toronto). Of the Pseudomonas species, $5 \%$ were resistant to each of piperacillin (Pipracil, Wyeth Ayerst Canada Inc, Montreal, Quebec), ciprofloxacin and ceftazidime, and none were resistant to the aminoglycosides. One pseudomonal isolate was resistant to both piperacillin and ceftazidime.

\section{DISCUSSION}

ICUs are often the target of surveillance programs and antimicrobial restrictions due to increased rates of bacterial resistance (2). Although it is difficult to make direct comparisons with other ICUs without detailed demographic information, the results of our study demonstrated a lower incidence of antimicrobial resistance among bacterial isolates of patients receiving parenteral antibiotics relative to reports from other American and Canadian sites (2,11-13). A similar study of resistance in Gram-negative bacilli in a 16-bed ICU in a Vancouver, British Columbia hospital demonstrated a much higher incidence of Gram-negative infections, 35\% of which were associated with beta-lactamase-mediated resistance (11). This study, however, included burn patients, which may have accounted for these results. Another study of 1939 Gramnegative isolates from 20 Canadian ICUs also demonstrated a higher incidence of resistance, particularly to beta-lactam antibiotics and ciprofloxacin (12). Our CrCU appeared to have a fewer number of more difficult to treat organisms such as pseudomonal isolates (13.5\%), compared with most ICUs where Pseudomonas species have been the primary pathogen, accounting for up to $28 \%$ of isolates $(4,12)$. In addition, we also had a very low incidence of MRSA isolates compared with other sites $(4,12,13)$. The findings in this study were reassuring in that the incidence of bacterial resistance among patients receiving antibiotics has remained low. Also, there were no outbreaks with multiply resistant organisms during the study, although the CrCU has experienced previous outbreaks, possibly due to the excessive use of broad-spectrum antibiotics (14).

The only study that has quantified microbial resistance rates relative to antibiotic use collected data on several antimicrobial-organism pairs from eight American hospitals (ICARE Project) (13). Data from the ICUs studied demonstrated a strong correlation between the use of ceftazidime and both ceftazidime-resistant $E$ cloacae and $P$ aeruginosa. Surprisingly, there was no relationship between resistance in $E$ cloacae and other third-generation cephalosporins. Furthermore, these relationships were not found in the non-ICU areas of the hospitals.

Several factors may have accounted for the low incidence of antimicrobial resistance in our CrCU. Seventy per cent of our population were surgical or trauma patients who tended to have fewer comorbid conditions and a shorter length of hospital stay. The average length of stay in all patients admitted to our CrCU during the study was only 4.7 days. These patients would, therefore, have had less opportunity to be exposed to the hospital environment and to other critically ill patients colonized with resistant organisms. Results of other studies have also demonstrated a reduction in morbidity and mortality in patients in surgical intensive care units relative to medical units (15).

The prudent use of antibiotics in the unit might have been another factor that contributed to the low level of antimicrobial resistance in our unit. Only $32 \%$ of patients admitted to the $\mathrm{CrCU}$ received antibiotics; in other centres, $83 \%$ of patients received antimicrobial agents (16). More important, thirdgeneration cephalosporins (ceftriaxone $255 \mathrm{~g}$, ceftazidime 265 g) and ciprofloxacin (49 g) were not used very often and no imipenem was prescribed during the study (Table 2). The moderate use of third-generation cephalosporins in the unit may 
have accounted for the lack of Gram-negative bacilli with ESßL during the period of surveillance. This is in contrast with the ICARE Project, which demonstrated higher use of thirdgeneration cephalosporins and higher microbial resistance rates. The presence of ESßL in our CrCU might also have been underestimated because routine testing for these betalactamases was only performed on cultures from sterile sites such as blood and CSF. At the time of this study, there were no guidelines for the detection of ESßL and only the E-test was used for screening. The absence of resistance to imipenem and the low rates of quinolone resistance were also likely a reflection of the limited use of these drugs.

While no attempt was made to assess formally the appropriateness of antibiotic prescribing patterns, antibiotic usage did appear to correspond to the clinical indications for which antimicrobial therapy was prescribed, as well as to the bacteria isolated from patients with infection. Active involvement of pharmacy, infectious disease and microbiology personnel may have also affected the appropriateness of antibiotic choice. Finally, the enforcement of strict infection control procedures in the $\mathrm{CrCU}$ may have influenced the low incidence of antibiotic resistance observed in the audit.

Although a low level of antimicrobial resistance was demonstrated, our study was limited by the lack of strict criteria and definitions for the diagnosis of specific infections. Furthermore, we assumed that the bacterial isolate recovered from the different sites corresponded to the clinical infections for each infective episode and did not represent colonization. For this reason, we may have overestimated the true infection

\section{REFERENCES}

1. Holmberg SD, Solomon SL, Blake PA. Health and economic impacts of antimicrobial resistance. Rev Infect Dis 1987;9:1065-78.

2. Archibald L, Phillips L, Monnet D, McGowan JE Jr, Tenover F, Gaynes R. Antimicrobial resistance in isolates from inpatients and outpatients in the United States: increasing importance of the intensive care unit. Clin Infect Dis 1997;24:211-5.

3. Donowitz LG, Wenzel RP, Hoyt JW. High risk of hospital-acquired infection in the ICU patient. Crit Care Med 1982;10:355-7.

4. Vincent JL, Bihari DJ, Suter PM, et al. The prevalence of nosocomial infection in intensive care units in Europe. Results of the European Prevalence of Infection in Intensive Care (EPIC) Study. JAMA 1995;274:639-44.

5. Stratton CW, Ratner H, Johnston PG, Schaffner W. Focused microbiological surveillance by specific hospital unit as a more sensitive means of defining antimicrobial resistance problems. Diagn Microbiol Infect Dis 1992;15:11S-8S.

6. Gaynes R. Antibiotic resistance in ICU's: a multifaceted problem requiring a multifaceted solution. Infect Control Hosp Epidemiol 1995; 16:328-30.

7. Chow JW, Fine MJ, Shlaes DM, et al. Enterobacter bacteremia: clinical features and emergence of antibiotic resistance during therapy. Ann Intern Med 1991;115:585-90.

8. Sanders WE Jr, Sanders CC. Inducible -lactamases: clinical and epidemiologic implications for use of newer cephalosporins. Rev Infect Dis 1988;10:830-8.

9. National Committee for Clinical Laboratory Standards. Methods for dilution antimicrobial susceptibility tests for bacteria that rates in the $\mathrm{CrCU}$. The present study only looked at the incidence of bacterial resistance with concurrent antibiotic utilization and did not evaluate whether this use accounted for the lower incidence. Finally, the overall prevalence of bacterial resistance in the $\mathrm{CrCU}$ was not determined, because we did not collect data on patients admitted to the unit who did not receive parenteral antibiotics. For this same reason, we could not compare resistance rates among those who received antibiotics and those who did not.

\section{CONCLUSIONS}

The results of this audit demonstrated that antimicrobial use within the $\mathrm{CrCU}$ appeared reasonable, and because of the low levels of bacterial resistance, no further recommendations regarding modifications of antimicrobial use were made. A combination of ongoing surveillance for bacterial resistance, monitoring of antibiotic utilization patterns and effective infection control practice may have contributed to this low rate. However, given the increasing reports of the rapid emergence of antimicrobial resistance in ICUs and the variability in resistance patterns in different parts of the hospital, we will continue focused, microbiological surveillance, and study whether appropriate monitoring of antibiotic usage and other factors affect the emergence of bacterial resistance.

ACKNOWLEDGEMENTS: The authors acknowledge WR Cornish, BScPhm and SAN Tailor, PharmD, members of the Antimicrobial Subcommittee of Sunnybrook and Women's College Health Sciences Centre, for their aid in the conceptualization of this audit.

grow aerobically, Approved standard M7-A2 (M100-S4). Villanova: NCCLS, 1993.

10. Jorgensen JH, McElmeel ML, Trippy CW. Comparison of inoculation methods for testing enterococci by using vancomycin screening agar. J Clin Microbiol 1996;34:2841-2.

11. Bryce EA, Smith JA. Focused microbiological surveillance and gram-negative beta-lactamase-mediated resistance in an intensive care unit. Infect Control Hosp Epidemiol 1995;16:331-4.

12. Bryce EA, Colby WD, Haket J, Poisson MW, Smith JA. Incidence and susceptibility of aerobic Gram-negative bacilli from 20 Canadian intensive care units: 1989-1993. Can J Infect Dis 1996; 7:34-40.

13. Monnet DL, Archibald LK, Phillips L, Tenover FC, McGowan JE Jr, Gaynes RP. Antimicrobial use and resistance in eight US hospitals: complexities of analysis and modeling. Infect Control Hosp Epidemiol 1998;19:388-94.

14. Batchelor C, Tsimidis K, Rachlis A, Louie M, Simor AE. Investigation of multiresistant Acinetobacter baumanii: use of molecular typing techniques. In: Program and Abstracts of the Twenty-first Annual Meeting of the Association for Practitioners in Infection Control, Cincinnati, Ohio, 1994. Am J Infect Control 1994;22:106.

15. Torres A, Aznar R, Gatell JM, et al. Incidence, risk, and prognosis factors of nosocomial pneumonia in mechanically ventilated patients. Am Rev Respir Dis 1990;142:523-8.

16. Dasta JF. Drug use in a surgical intensive care unit. Drug Intell Clin Pharm 1986;20:752-6. 


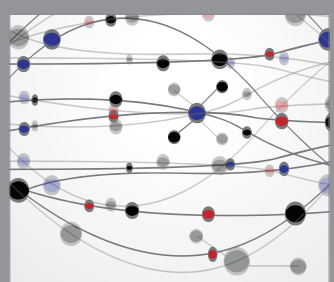

The Scientific World Journal
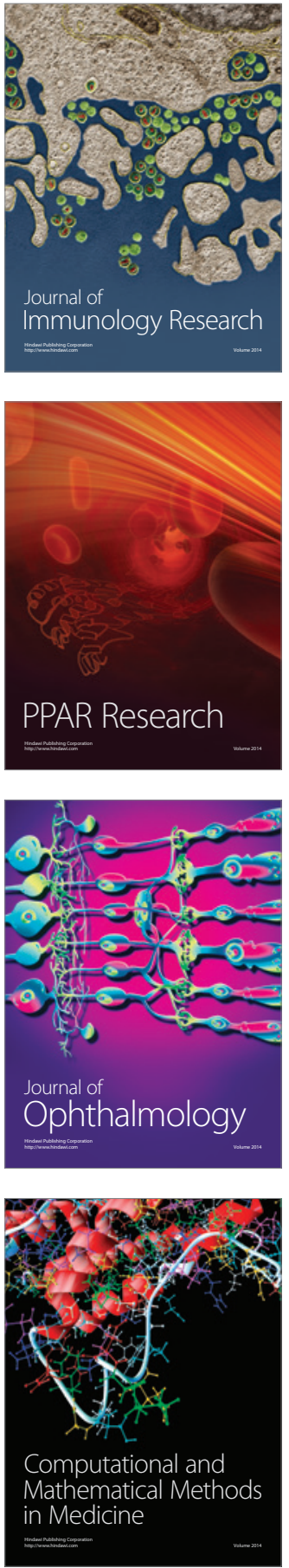

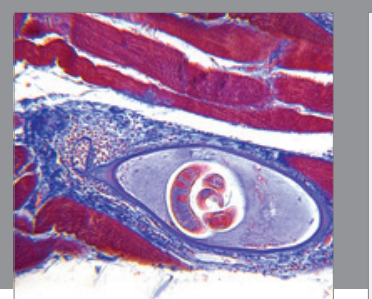

Gastroenterology Research and Practice

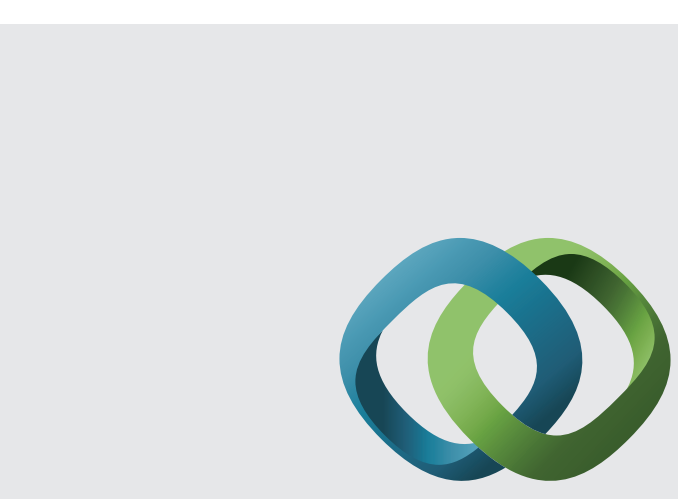

\section{Hindawi}

Submit your manuscripts at

http://www.hindawi.com
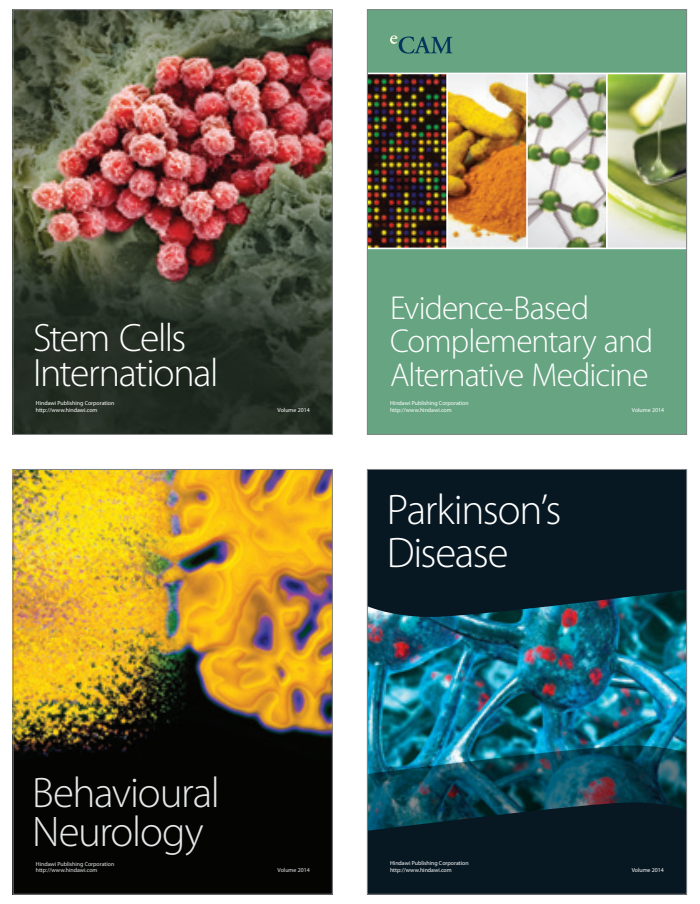
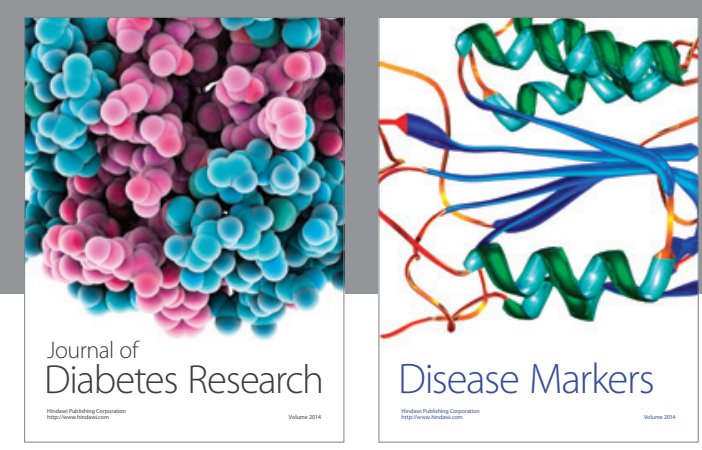

Disease Markers
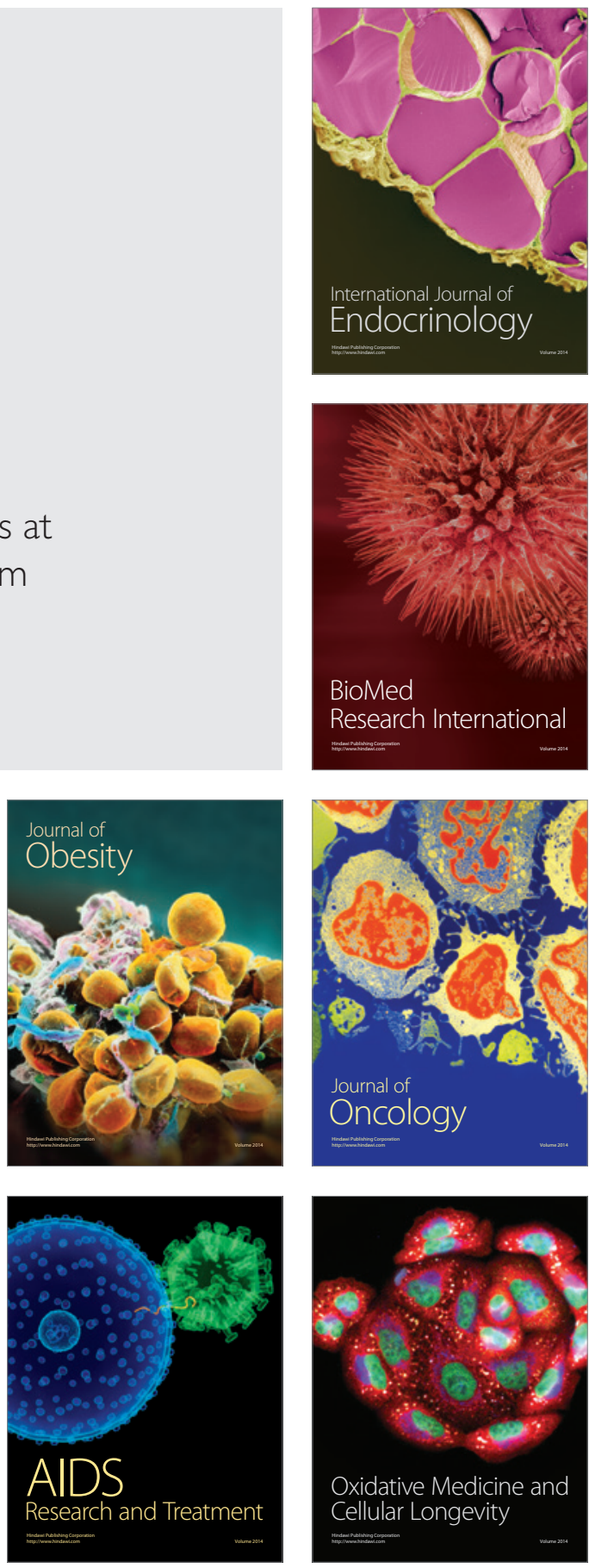